\title{
A NOVEL HANDOVER ALGORITHM FOR LTE BASED MACRO-FEMTO HETEROGENEOUS NETWORKS
}

\author{
Bhargavi D K ${ }^{1}$ and Dr.Vijaya Prakash A M ${ }^{2}$ \\ ${ }^{1,2}$ Dept. of Electronics \& Communication, BIT, Bangalore, India
}

\begin{abstract}
This paper presents a novel Handover mechanism to minimize target Femto Access Points (FAPs) list during macro-to-femto and femto-to-femto handover in LTE based Macro-Femto Heterogeneous Networks. HandOver is the procedure that transfers an ongoing call from one cell to another as the users move through the coverage area of cellular system. The femtocell has a limited coverage area (15 meters to 30 meters), and subsequently, HandOver is decided based on mobile User Equipment (UE) speed for handover between macrocell-to-femtocell and femtocell-to-femtocell. In the existing networks, for handover procedure, serving Base Station will decide the cell selection based on mostly the signal strength of the neighbouring femtocells. This information is provided by the Measurement Reports sent by the corresponding UE. In this paper, we present a novel handover management scheme which considers both the Received Signal Strength and Cell Load of the target Femtocells for making the Handover decision. This proposed scheme for macro-femto heterogeneous network reduces the number of target FAPs to avoid handover to overloaded femtocells and also reduce the number of handovers.
\end{abstract}

\section{KEYWORDS}

Femtocells; HandOver; Soft Handover; Hard Handover; Hand-in; Hand-off; Femto Access Points; (e)NB and $H(e) N B$.

\section{INTRODUCTION}

The femtocell networks are one of the most promising technologies to meet the demand of the ever increasing wireless applications for the future wireless communications. Higher data rates in wireless networks have always been a demand of modern day users. Voice over IP (VoIP), ultra broadband access, real-time and streamed multimedia, gaming services and many other such activities require increased bandwidth for flawless communication.

Many new technologies are being introduced with higher data rates among which Femtocells are most popular ones in recent times with more and more deployments in real time. The most important advantages femtocell networks are offloading huge traffic from the expensive cellular networks; less deployment cost; and use of same frequency as that of cellular networks. Thus, the deployment of femtocells in large scale is the ultimate goal for this technology.

The large and dense scale deployment of femtocells suffers from several challenges. Handover is one main challenge among several other challenges. Three types of handovers occur in dense femtocells environment; macrocell to femtocell handover also called as Hand-in, femtocell-tomacrocell handover and femtocell-to-femtocell handover also called as Hand-off. Femtocell-toMacrocell handover does not suffer from additional challenges. However, the macrocell-to-

DOI : $10.5121 /$ vlsic. 2015.6403 
femtocell and femtocell-to-macrocell handovers face some difficulties including the selection of appropriate femtocell for handover and the optimal neighbour femtocell list for the handover. In this paper, we address the issue of handling appropriate neighbour femtocell list for Hand-in and Hand-off.

Femtocell networks are used for small coverage area, $(15 \mathrm{~m}$ to $30 \mathrm{~m})$ and UE's supported are 4 to 5 in residential settings. Femtocell Base Station [14] will support Self-Organizing Networks (SON) capability to enable plug and play model where a subscriber will be able to purchase Femtocell and then simply plug it into the broadband modem to receive services without any operator intervention. Usage of femtocells offers multiple advantages for both subscribers and operators.

A cognitive femtocell network comprises of distinct kinds of Base Stations (BS): femto BS, macro BS and also contains femto group. Femto group contains the set of femtocells which covers the specific region. In a cognitive femto network, due to the small coverage radius and some of the cells being open access, interference caused is higher and hence, handover is one of the most challenging issues.

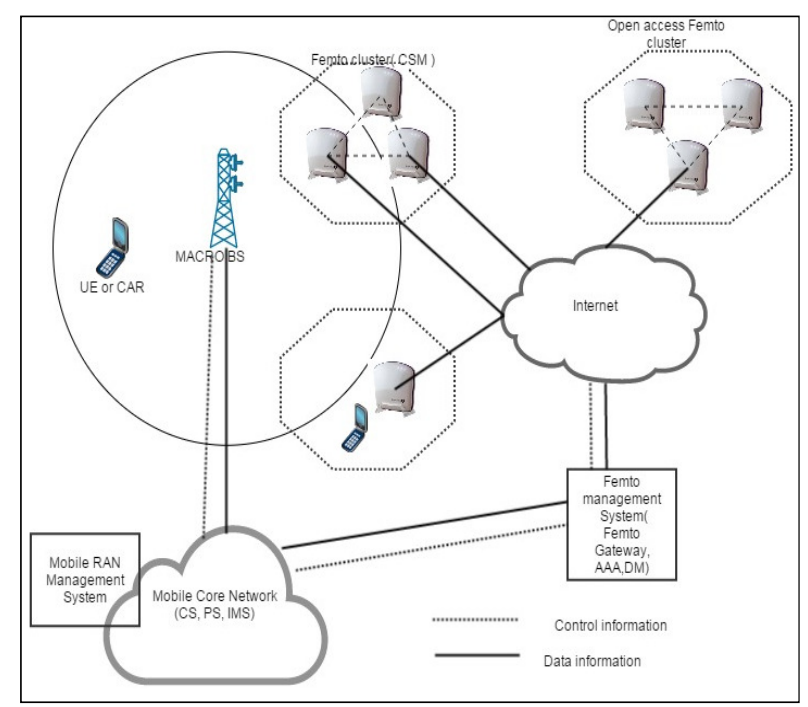

Fig. 1. Cognitive Femtocells Network

Cell selection is an important function in femto-to-femto handover scenario. Handover management aims to find the accurate target femtocell while reducing the number of unnecessary handovers [6], avoiding overloaded femtocells and choosing appropriate target FAP. Femto subscribers can access the Femto Network by three modes [12]: Open Access Mode, Closed Access Mode and Hybrid Access. In open access mode, access is provided to all subscribers, and it operates as a normal macrocell base station. In closed access mode, the femto will provide the services only to configured UEs. In hybrid mode, the femto allows unauthorized UEs to access limited services such as voice service etc.

As mentioned already, the cognitive femto network's architecture model [1] is portrayed in Fig. 1. In this architecture model, we assume that the Femto Device Management System (FDM) and mobile Radio Access Network (RAN) Management System trade information periodically. When a UE moves between one femto to another femto or macro Base Station (MBS) coverage area, UE should support handover connection establishment and update its location [10-11]. In this Femtocell network, the femtocell-to-femtocell handover is very difficult. This handover scenario is very important because the femtocells are using very small coverage area like building, airports, shopping center, railway station, etc. 
The handover process has two types of failure conditions [4], they are: Too Late Handover (TLH) and Too Early Handover (TEH). Fig. 2 demonstrates that handover failure due to radio link failure condition. In case of TEH as shown in Fig. 2(a), when a UE is moving from MBS towards femtocell, where MBS is a serving cell and target cell femto, it is too early to handover. In this case, due to signal strength of target cell being low causes radio link failure or Ping-Pong effect in handover procedure. Then UE will re-establish radio link connection to serving cell (MBS-Macro Base Station). In case of TLH as shown in Fig. 2(b), here also radio link fails from the serving femto cell due to very low signal strength in the late handover procedure. Then UE will establish radio link connection with target cell (MBS).

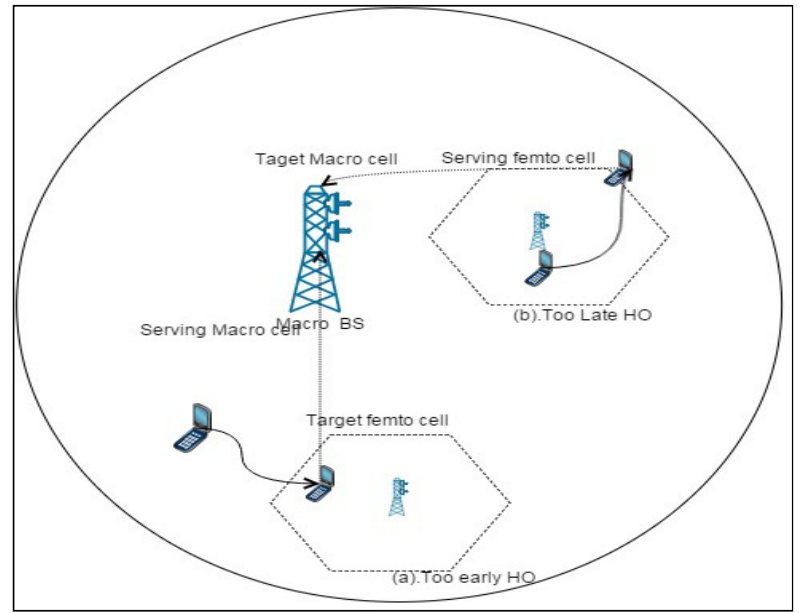

Fig. 2. Too Early Handover and Too Late Handover Depiction

In the wake of portraying a few key ideas, rest of the paper is composed as follows: we describe the LTE based femtocell architecture model in section II. In section III, we present proposed algorithm for minimizing target FAPs list and finally section IV gives the conclusion and remarks about the paper.

\section{LTE BASED FEMTOCELLS NETWORK ARCHITECURE}

This section will explain the architecture of LTE based femtocell network and its components from the handover perspective [3] as shown below in Fig. 3. This Figure contains femtocell or Femto Access Point (FAP), Home (e) Node B (H(e)NB) in Macro-Femto Heterogeneous network [5] and it is connected to internet by using copper/fiber wire. Ex: DSL/FTTH/WiMAX etc. All $\mathrm{H}(\mathrm{e}) \mathrm{NBs}$ or FAPs are connected to the Mobile Switching Centre (MSC) and the Packet Data Network (PDN) through femto gateway called H(e)NB-GW and all macrocell ((e)NB) base stations are connected to (e)NB Gateway ((e)NB-GW) for control signalling. These two gateways are designed for providing functionality to handle supported user data, PDN and to provide the routing and forwarding between (e)NBs or $\mathrm{H}(\mathrm{e}) \mathrm{NBs}$. And it also acts as the mobility anchor during handovers between LTE and other 3GPP systems [7, 9]. MBS is connected to Mobility Management Entity (MME) system as shown in Fig. 3. MME, the main control device/node for 3GPP-LTE access network provides the control plane functionality for UE between LTE and other access networks. MME also takes care of choosing the appropriate Security Gateway (SeGW) for a UE and provides authentication to them. A H(e)NB-GW is used to provide the interface scalability and supports larger number of $\mathrm{H}(\mathrm{e}) \mathrm{NBs}$. It also works as a concentrator for the control plane. 
International Journal of VLSI design \& Communication Systems (VLSICS) Vol.6, No.4, August 2015

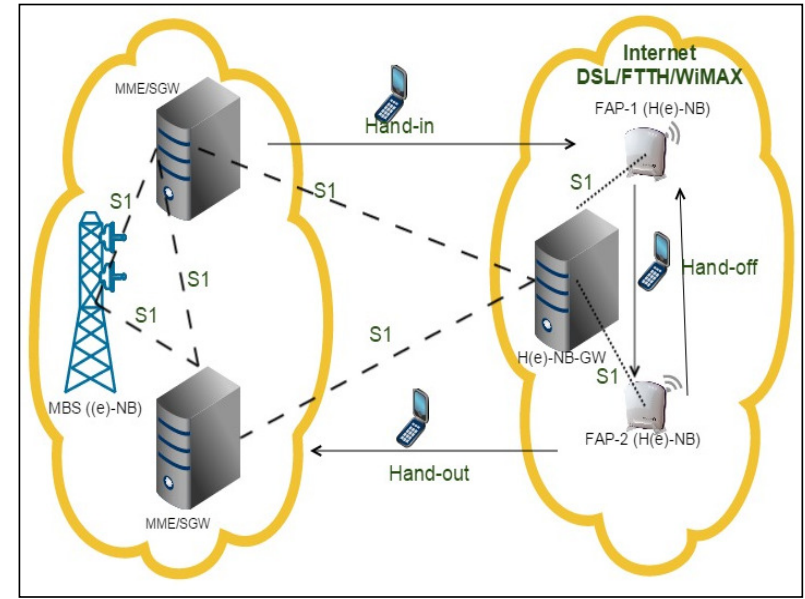

Fig. 3. LTE based Macro-Femto Hetnet Architecture

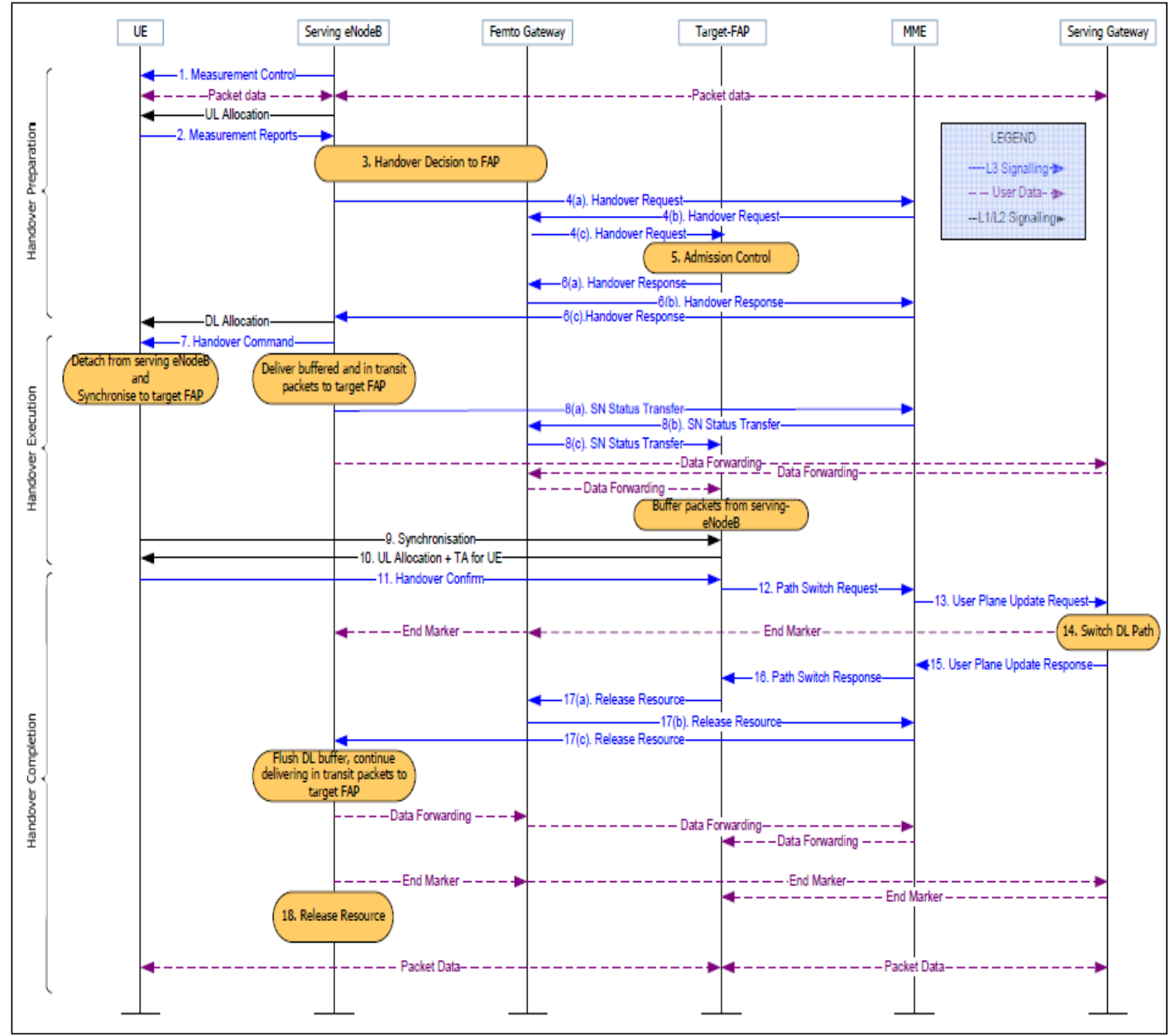

Fig. 4. Hand-in signalling flow (Macrocell to Femtocell)

\section{A. Handover Scenario's in Macro-Femto Heterogeneous Network}

In femtocell networks several research works are going on for the handover. As per standards, there are three types of handovers: Hand-in, Hand-out and Hand-off (Inter-femto) handovers [7]. 


\section{Hand-in:}

This kind of handover happens when a UE is moving from macrocell ((e)NB or MBS) coverage area into FAP $(\mathrm{H}(\mathrm{e}) \mathrm{NB})$ coverage area and it is also the most complex handover scenario compared to other handovers [8]. For this situation Mobile Station (MS) or UE needs to select the right target FAP among the hundreds of FAPs list. The signalling flow diagram for Hand-in scenario in LTE is shown in Fig. 4.

\section{Hand-out:}

In this handover scenario UE switches from serving FAP to macrocell (e)NB [2]. This scenario does not pose many problems as compared to the Hand-in procedure. Because the target cell is macro, there is no option other than macrocell network and its procedure seems like handover from one base station to another base station. In this handover, one of the issues is to keep the handover time to be very small.

At the point of handover, signal strength of the target macrocell (e)NB must be stronger or greater than the serving femtocell $\mathrm{H}(\mathrm{e}) \mathrm{NB}$, and then UE will directly get connected to it. There is no other complex interface calculation and authentication as in case of the Hand-in procedure.

\section{Hand-off:}

This scenario also called as Inter-femto handover [8], because the handover will happen between a FAP to another FAP within a group. In this scenario, UE switches from one femtocell coverage area to another femtocell coverage area. In the Hand-off scenario, UE will be facing more number of target FAPs list, when serving H(e)NB is out of service. It is similar to Hand-in scenario.

\section{B. Handover Procedure and Flow Diagram}

This section describes the steps involved in handover procedure. The handover procedure contains three principle steps, they are: Preparation, Execution and Completion (Fig. 4).

- Handover Preparation: In this step, before UE switches from serving (e)NB to target FAP $(\mathrm{H}(\mathrm{e}) \mathrm{NB})$, it needs to make some preparation. The main messages and process for these steps are as shown in Fig. 4 and are explained below:

1. Scan and send Measurement Report (1-2 ): The serving (e)NB periodically sends the measurement request to UE, which scans the neighbor cells and sends the Measurement Report (MR) to serving (e)NB.

2. Handover Decision (3-4): Serving (e)NB takes the handover decision based on the MR received from UE. Internally serving (e)NB sends handover request to MME as demonstrated in message 4(a) and MME will send request to (e)NB gateway and gets response from it.

3. Admission Control (5-6): The target FAP will perform the admission control depending on QoS information and prepares handover with L1/L2.

4. Handover Command (7): The serving macrocell (e)NB sends the handover command to UE.

- Handover Execution: This part deals with the execution of the handover command in UE and detaching from serving (e)NB to synchronize to the new target $\mathrm{H}(\mathrm{e}) \mathrm{NB}$ (8-10); Also, UE performs the synchronization with new target cell and accesses the target cell.

- Handover Completion (11-18): This is the last step in handover procedure that is finished by sending the handover confirm message and serving gateway switches the downlink path 
to target FAP. This is achieved by target $\mathrm{H}(\mathrm{e}) \mathrm{NB}$ sending switch path messages to MME as demonstrated in Fig. 4 (11-16). The serving (e)NB releases resources like radio bearer, while subsequently target FAP sends downlink packet data (17-18).

\section{PROPOSED HANDOVER ALgORITHM}

After gaining insight into several key ideas about handover process and considering that hand-in and hand-off scenario causes fundamental issue with target FAPs list, this section describes the concept of proposed pseudo code for optimization algorithm to minimize target FAPs list. Consider in Fig. 5(a), initially the UE is connected to (e)NB or $\mathrm{H}(\mathrm{e}) \mathrm{NB}$. When the serving (e) $\mathrm{NB} /(\mathrm{H}(\mathrm{e}) \mathrm{NB})$ signal strength decreases, hand-in (or hand-off) will take place to appropriate target $\mathrm{H}(\mathrm{e}) \mathrm{NB}$. UE will scan the neighbour target FAPs and sends their information to serving (e)NB periodically in the measurement report. Fig. 5(b) shows the blue colour circle of area of neighbour FAP candidates. We propose the algorithm to minimize the number of these target FAP candidates with respect to UE. Section III.A defines the concept of algorithm and III.B describes the pseudo code.

\section{A. Handover Algorithm Concept}

The handover procedure consists of four main steps:

1. Scanning and sending Measurement Reports of neighbour Femtocells to serving Base Station.

2. Selecting the appropriate target FAP for Handover.

3. Execution of Handover Procedure.

4. Completion of Handover.

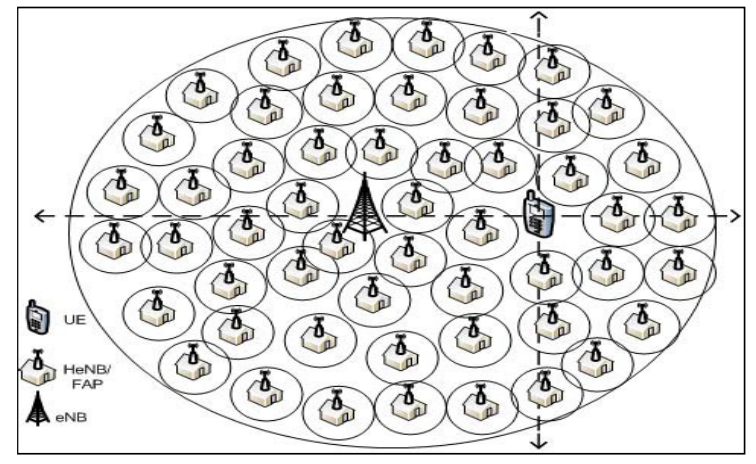

Fig. 5(a). UE connected to a (e)NB

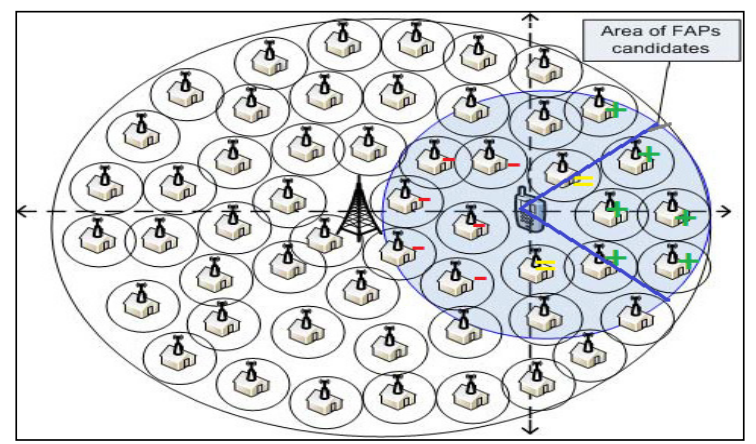

Fig. 5(b). UE Initiates handover measurement and scan 
In the scanning and measurements step, serving cell (femto or macro) will periodically send a measurement signal request to the UE which will send the feedback with a measurement report. The measurement reports contain the speed of UE and Received Signal Strength (RSSI) of neighbour cells. Assuming that the load capacity of neighbour Femtocell is provided by the Gateway to the Base Station, we propose our algorithm to select the best target FAP.

Among the available neighbour FAPs, the RSSI and the cell load will be tabulated for each FAP. Based on the most suitable FAP with higher RSSI and lower cell load, best FAP will be selected as target FAP for handover. In Fig 5 (b), green FAPs denote the FAPs for which RSSI is increasing and the red FAPS denote the decreasing RSSI values with respect to UE. After the target cell is selected, serving cell will handover the UE to the target cell. In handover completion step, serving cell will terminate the connection to UE and MME will switch the path of downlink signal to target cell.

\section{B. Handover Flow Control Procedure}

Before establishing the connection with the target FAP (Tar-H(e)NB), a handover control procedure will be triggered based on the following criteria:

1) The signal level of suitable Target-H(e)NB (FAP) should be higher than a fixed threshold.

2) Cell load of the target FAP must be lower than $3 / 4^{\text {th }}$ of the Cell Capacity.

3) A table will be created for the FAPs with RSSI higher than threshold value.

4) Select the FAP with best RSSI value and optimum cell load.

5) Execute Handover Procedure.

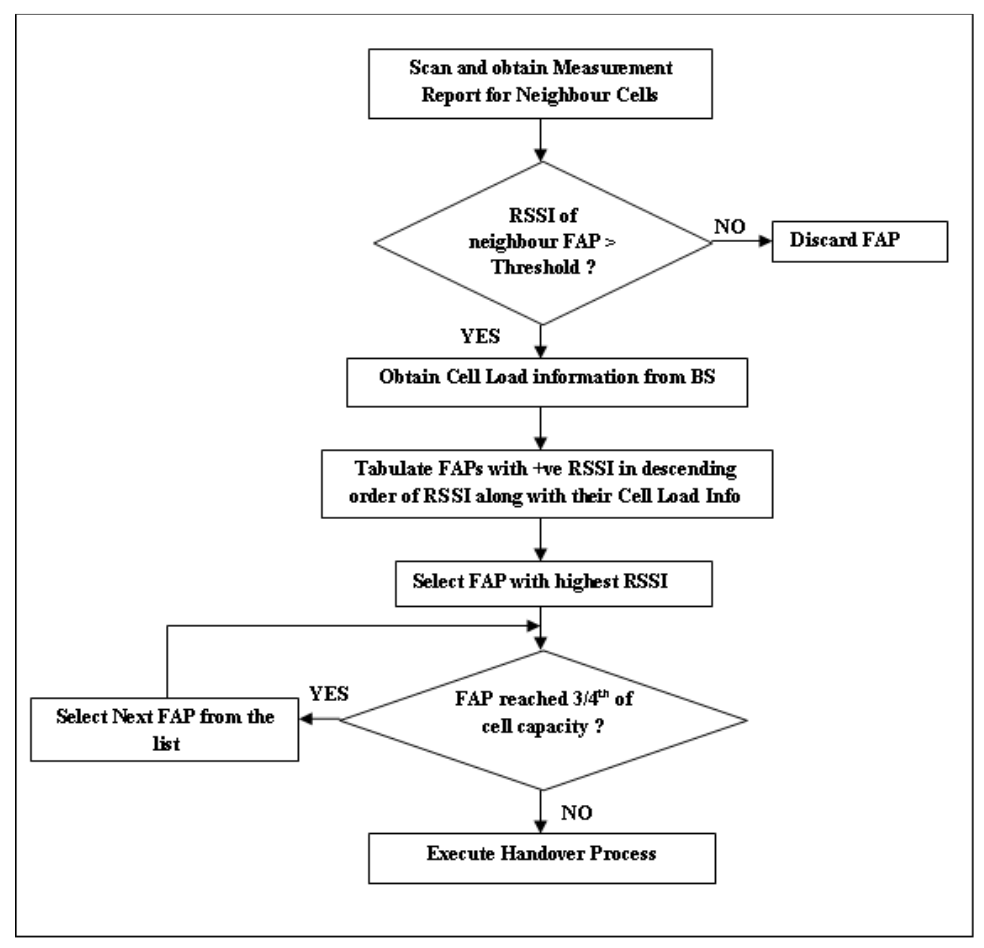

Fig. 6. Handover Flow Control Procedure 
The Handover Flow Procedure is shown in figure 6. The complete process depends on the RSSI value of the neighbour FAPs along with their cell load. The best FAP with optimum RSSI and cell load is selected and the Handover Process is executed.

\section{A. Pseudo code for the Proposed Algorithm}

The Pseudo code for minimizing target FAPs is demonstrated in below Fig. 8. After finding target FAPs (area of FAP candidates in Fig. 5(b)), serving cell finds out whether the RSSI of the FAP is greater than threshold or not and the cell load is less than $3 / 4^{\text {th }}$ of the cell capacity or not. By using this concept serving cell will choose the proper target FAP and avoids more number of handovers and unsuccessful handovers.

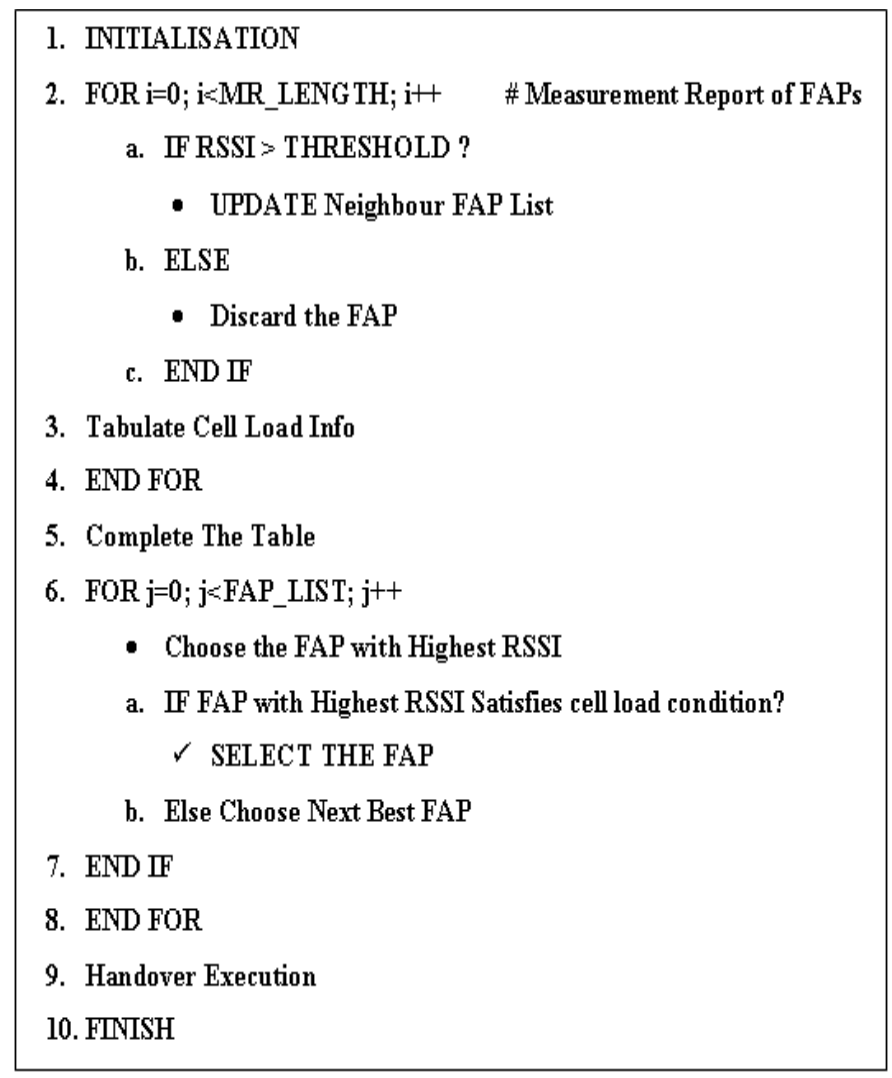

Fig. 7. Pseudo code for the Proposed Algorithm

The UE can be authorized to access the Target - H(e)NB only if the number of UEs connected to the Tar-H(e)NB would not exceed $3 / 4^{\text {th }}$ of the maximum number of permitted users according to the Tar-H(e)NB access mode. As illustrated in Fig.7, the first part focuses on sending the measurement reports sent by the UE. The second part concentrates on computing optimized list of target FAP candidates by using the proposed algorithm. The last part consists of the handover execution and releasing procedure.

\section{CONCLUSION}

For the femto-to-femto handover in the dense femto network deployment, one of the most important considerations is the small number of neighbor femtocells in the neighbor femtocell list.The lesser number of entries in the neighbor Femto list reduces the power consumption for scanning many FAPs and also reduces the MAC overhead. 
In this paper, we have proposed a new algorithm to minimize the target FAPs and reduce number of handovers by choosing the best target FAP among the neighbour FAPs list. During the handover procedure, we choose the target FAP with optimum RSSI value and optimum Cell Load to avoid the handover failure and frequent subsequent handovers. The handover takes place specific to a UE as identified in the FAP's list.

Limitation is such that, it requires the extra processing than the formal handover and also Femto should maintain a separate table for each UE attached to it. In future it can be modified to maintain unified table for all the UEs attached to the femto.

\section{REFERENCES}

[1] Nhu-Dong Hoang, Nam-Hoang Nguyen and Keattisak Sripimanwat "Cell Selection Schemes for Femtocell-to-Femtocell Handover deploying Mobility Prediction and Downlink Capacity Monitoring in Cognitive Femtocell Networks", IEEE Region 10 Conference, Oct-2014.

[2] Ahlam Ben Cheikh1,2, Mouna Ayari1,2, Rami Langar2, Guy Pujolle2, and Leila Azouz Saidane1,"Optimized Handover Algorithm for Two-tier Macro-Femto Cellular LTE Networks", WiMob-IEEE 9th International Conference, Oct-2013.

[3] Shayea. I et.al, "Advanced handover techniques in LTE- Advanced system", International Conference on Computer and Communication Engg, July-2012.

[4] Hyung-Deug Bae, ByungHan Ryu and Nam-Hoon Park "Analysis of Handover Failures in LTE Femtocell". Australian Telecommunication Networks and Applications Conference (ATNAC), Nov 2011.

[5] Femto Forum, "The Femto Forum: H(e)NB (LTE Femto) Network Architecture", www.femtoforum.org, May 2011.

[6] H. Zhang, X. Wen, B. Wang, W. Zheng and Y. un, "A Novel Handover Mechanism between Femtocell and Macrocell for LTE based Networks", pp. 228 231, Second International Conference on Communication Software and Networks, ICCSN, Feb-2010.

[7] Ardian Ulvan, Robert Bestak and Melvi Ulvan, "Handover Scenario and Procedure in LTE-based Femtocell Networks", UBICOMM: The Fourth International Conference on Mobile Ubiquitous Computing, Systems, Services and Technologies, Oct-2010.

[8] 3GPP TS 23.009, "Handover Procedures," March 2009.

[9] 3GPP TS 36.300-870, "3rd Generation Partnership Project", 2009.

[10] H. Kwak, P. Lee, Y. Kim, N. Saxena and J. Shin, "Mobility Management Survey for Home-eNB Based 3GPP LTE Systems", Journal of Information Processing Systems, Vol.4, No.4, December 2008.

[11] V. Chandrasekhar, J. Andrews and A. Gatherer, "Femtocell networks: A Survey, IEEE Communications Magazine", September 2008.

[12] Mostafa Zaman Chowdhury and Yeong Min Jang," Handover Control for WCDMA Femtocell Networks", Sept.2008.

[13] 3GPP TR R3.020, "Home (e) Node B: Network Aspects," Sept. 2008.

[14] Ayaskant Rath and Shivendra Panwar, "Fast Handover in Cellular Networks with Femtocells", May 2008. 